\title{
BMJ Open Prevalence of Gram-negative bacteria in ventilator-associated pneumonia in neonatal intensive care units: a systematic review and meta-analysis protocol
}

\author{
Yousef Erfani, ${ }^{1}$ Arezoo Rasti, ${ }^{2}$ Leila Janani ${ }^{3}$
}

To cite: Erfani Y, Rasti A, Janani L. Prevalence of Gramnegative bacteria in ventilatorassociated pneumonia in neonatal intensive care units: a systematic review and metaanalysis protocol. BMJ Open 2016;6:e012298. doi:10.1136/ bmjopen-2016-012298

- Prepublication history and additional material is available. To view please visit the journal (http://dx.doi.org/ 10.1136/bmjopen-2016012298).

Received 14 April 2016 Revised 15 September 2016 Accepted 19 September 2016

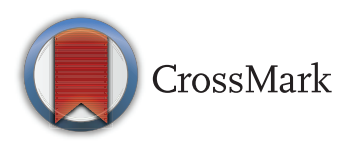

${ }^{1}$ Department of Medical Laboratory Sciences, School of Allied Medical Sciences, Tehran University of Medical Sciences, Tehran, Iran ${ }^{2}$ Oncopathology Research Center, Iran University of Medical Sciences, Tehran, Iran

${ }^{3}$ Department of Biostatistics, School of Public health, Iran University of Medical Sciences, Tehran, Iran

Correspondence to Dr Yousef Erfani; yerfani@sina.tums.ac.ir

\section{ABSTRACT}

Introduction: Ventilator-associated pneumonia (VAP) is a common and potentially lethal problem among mechanically ventilated neonates in neonatal intensive care units (NICUs). The main pathogenic bacteria of VAP in NICUs are Gram-negative pathogens, which show a general decline in sensitivities to commonly used antibiotics, but their true prevalence is not known.

Methods and analysis: We aim to provide a systematic review of studies measuring the prevalence of Gram-negative bacteria in VAP in NICUs. We will search PubMed, SCOPUS, EMBASE and the ISI Web of Science, as well as the Google Scholar search engine with no restriction on language. Full copies of articles will be identified by a defined search strategy and will be considered for inclusion against predefined criteria.

Study selection and data extraction will be performed by 2 independent reviewers. Statistical analysis will include the identification of data sources and documentation of estimates, as well as the application of the randomeffects and fixed-effects meta-analysis models. This will allow us to aggregate prevalence estimates and account for between-study variability in calculating the overall pooled estimates and $95 \% \mathrm{Cl}$ for the prevalence of Gram-negative bacteria in VAP in NICUs. Heterogeneity will be evaluated using the $\mathrm{I}^{2}$ and $\chi^{2}$ statistical tests to determine the extent of variation in effect estimates due to heterogeneity rather than chance. Publication bias and data synthesis will be assessed by funnel plots and Begg's and Egger's tests using STATA software V.13. This systematic review protocol was prepared according to the Preferred Reporting Items for Systematic reviews and Meta-Analyses Protocols (PRISMA-P) 2015 Statement.

Ethics and dissemination: No ethical issues are predicted. These findings will be published in a peerreviewed journal and presented at national and international conferences.

Trial registration number: CRD42016036048.

\section{INTRODUCTION}

Ventilator-associate pneumonia (VAP) is pneumonia in mechanically ventilated patients, and develops after the patient has

\section{Strengths and limitations of this study}

- This is the first attempt as a systematic review to summarise the prevalence of Gram-negative bacteria in ventilator-associated pneumonia (VAP) in neonatal intensive care units (NICUs).

- We will include observational studies that used the US Centers for Disease Control and Prevention $(\mathrm{CDC})$ and National Healthcare Safety Network (NHSN) definitions.

- The study screening, data extraction and the risk of bias of the current study will be assessed independently by two researchers.

- This study could potentially help policymakers and guideline developers in the management of neonates with VAP in NICUs.

- This review is restricted to a neonatal population ( $<1$ year of age).

- This review will be limited by the quality and heterogeneity of the primary studies.

been placed on mechanical ventilation (MV) for at least 48 hours. ${ }^{1-4}$ MV is an essential feature of modern neonatal intensive care unit (NICU). Unfortunately, it is associated with a substantial risk of VAP. ${ }^{5}$ Tracheal intubation is associated with a 3-21-fold risk of developing pneumonia. ${ }^{6}$ VAP is the second most common hospital-acquired infection among paediatric and NICU patients. ${ }^{3}{ }^{4}$ The range of VAP incidence density rates in children and neonates is large. Rates have been reported as low as $1 / 1000$ ventilator days and as high as $63 / 1000$ ventilator days. The incidence follows a geographical distribution and depends on the type of hospital and the country's income level. ${ }^{7}$

VAP is associated with increased duration of hospital stay resulting in high morbidity and mortality among NICU patients. ${ }^{8-10}$ Surveillance studies of nosocomial infections in NICU patients indicate that pneumonia comprises $6.8-32.3 \%$ of nosocomial infections in this setting. ${ }^{11-13}$ 
Many factors predispose infants to acquiring VAP; infants mechanically ventilated in the NICU are at a particularly high risk of developing VAP because of poor host factors, severe underlying diseases, prolonged use of MV, inadequate pulmonary hygiene, and extensive use of invasive devices and procedures. ${ }^{14-17}$ In addition, poor nutritional state and hypoalbuminaemia contribute to the development of VAP in neonates. ${ }^{6}$ The aetiological agent of VAP may differ according to the length of hospital stay, comorbid conditions and exposition of antimicrobials. ${ }^{18}$ Since many premature babies require MV, VAP has become a major challenge in NICUs. VAP has a large influence on neonatal survival, morbidity, hospital costs and duration of NICU stay. ${ }^{4} 1920$

Neonates have unique characteristics predisposing them to nosocomial infections. These patients' immature immune systems place them at an increased risk of infection. ${ }^{21}$ Skin and mucous membranes are more permeable and are less effective barriers. ${ }^{22}$ The pathogenesis of VAP involves two processes: bacterial colonisation of the aerodigestive tract and aspiration of contaminated oral secretions into the lower airways, as endotracheal tubes used to ventilate neonates are not cuffed. ${ }^{23}$

The clinical criteria for the diagnosis of VAP have been established by the National Healthcare Safety Network (NHSN) and the US Centers for Disease Control and Prevention (CDC). However, it should be noted that no gold standards currently exist for the diagnosis of VAP in neonates. ${ }^{6-10}$ Microbial diagnosis of VAP is based on the culture of samples obtained from the lower respiratory tract by tracheal aspirate, which is considered a less-invasive method with an acceptable diagnostic accuracy. ${ }^{24}$ Understanding the microbiology of VAP is critical for choosing an empirical antibiotic therapy; however, lack of a specific definition and difficulties obtaining uncontaminated samples of the lower respiratory airway render microbiological diagnosis and aetiological treatment extremely difficult. ${ }^{25}$

The most commonly isolated causative organisms are Gram-negative bacteria which show a general decline in sensitivities to commonly used antibiotics. ${ }^{26}$ Aerobic Gram-negative bacilli account for more than $60 \%$ of VAP cases. However, some investigators have reported that Gram-positive bacteria have become increasingly more common, with Staphylococcus aureus being the predominant isolate. ${ }^{27}$ The most common pathogens isolated in the neonatal population are Pseudomonas aeruginosa and $S$. aureus. ${ }^{7} 28$ However, isolation of other microorganisms such as Klebsiella pneumoniae and Escherichia coli has also been reported. Isolated Acinetobacter strains are practically resistant to all generally prescribed antibiotics. ${ }^{2} 28$ 30-33

Prevalence studies of Gram-negative bacteria in VAP in NICUs are even sparser. Badr et $a \hat{l}^{34}$ revealed that Gram-negative bacteria were isolated from the majority of patients with VAP $(68.6 \%)$, with Klebsiella predominating the positive culture $(34.3 \%)$. Koksal and colleagues found that Acinobacter was the predominating causative agent, whereas Petdachai reported that Pseudomonas was the most common organism isolated. ${ }^{35} 36$

The prevalence of Gram-negative bacteria in developing VAP in NICUs has been reported, and estimates range from $60 \%$ to $97 \%$ with Pseudomonas, Klebsiella and Acinobacter organisms predominating the positive cultures. $^{2} 92628303134$

It is controversial to what extent we can rely on epidemiological results for the prevalence of Gram-negative bacteria in VAP in NICUs. This calls for caution when the epidemiological data for the prevalence are interpreted, and indicate that it is important to attempt to understand what underpins the variation. Accurate estimates of the true prevalence of a causative organism are of value in planning diagnostic and intervention services.

It is possible that study design, the definition of VAP and differences related to the included participants and sample collection methods explain the inconsistencies in the currently available studies.

We propose to conduct a systematic review and meta-analysis to investigate the prevalence of Gramnegative bacteria in VAP in NICUs.

\section{Objectives}

The primary objective of this review is to conduct a systematic review and meta-analysis to estimate the prevalence of Gram-negative bacteria in VAP in NICUs. The secondary objectives of this review are: (1) to find the most commonly isolated causative Gram-negative bacteria in VAP in NICUs and its antibiotic susceptibility pattern, (2) to estimate pooled mean VAP rate (VAPs per 1000 mechanical ventilator days) and (3) to evaluate the risk factors of VAP in NICUs. This review will complement the findings of existing published reviews. ${ }^{7} 1025$

\section{Review questions}

This systematic review will be guided by the following research questions:

1. What is the prevalence of Gram-negative bacteria in VAP in NICUs in different countries?

2. What are the most commonly isolated causative Gram-negative bacteria in VAP in NICUs?

3. What are the antibiotic susceptibility patterns of isolated causative Gram-negative bacteria in VAP in NICUs?

\section{METHODS}

The Preferred Reporting Items for Systematic reviews and Meta-Analyses for Protocols 2015 (PRISMA-P 2015) have been used for preparing and reporting the protocol of this systematic review. ${ }^{37}$ In addition, the PRISMA flow diagram will be employed to describe the flow of information through the different phases of this systematic review. ${ }^{38}$ The protocol of this systematic review has been registered in PROSPERO 2016 (registration number CRD42016036048). 


\section{Inclusion criteria}

Observational studies (cross-sectional, case-control and cohort) describing the prevalence of Gram-negative bacteria in VAP in NICUs will be included. We will consider published articles in any languages, with full English abstracts.

\section{Exclusion criteria}

1. Duplicate publications of the same material. When the study has been published in more than one journal, the most recent and complete version will be used.

2. Narrative reviews, opinion pieces, letters or any other publications lacking primary data and/or explicit method descriptions.

\section{Search methods for identification of studies \\ Electronic searches}

We will search PubMed, SCOPUS, EMBASE and the ISI Web of Science, as well as the Google Scholar search engine with no language restrictions until 15 May 2016.

Keywords: Systematic Review, Ventilator Associated Pneumonia, NICU, Gram Negative bacteria.

\section{PubMed search strategy}

MeSH tags were found in PubMed. The details of the PubMed database search syntax are provided below.

(Prevalence OR incidence OR frequency OR outbreaks OR occurrence OR epidemiology OR epidemiologic studies OR 'population-based') AND ('ventilator-associated pneumonia' OR pneumonia) AND 'Gram negative' AND (bacteria OR microorganism OR pathogens).

The search syntax will be modified in other databases.

\section{Other resources}

Reference lists of relevant primary studies, reviews and key journals will be searched for additional studies.

\section{Selecting studies for inclusion}

Full-text articles identified by the search that will potentially meet inclusion criteria based on the title and abstract will be obtained for data synthesis. Studies will be screened against predefined inclusion and exclusion criteria. Two authors will be assigned to evaluate and appraise the results of the searches, based on the title and abstract. The reviewers will then either mark the studies as included or excluded. Once all the studies have been reviewed independently, the reviewers will together compare their evaluations; discrepancies will be discussed and, if necessary, a third reviewer will be called to resolve any disagreements.

\section{Data extraction and management}

A data extraction form will be developed, and study data will be independently assessed and extracted by two reviewers (YE and $\mathrm{LJ}$ ).

The following data will be extracted from all the included studies:
1. Study characteristics (author, year of publication, language of publication, country, study design, setting, locations, criteria for sample selection and sample size, diagnostic criteria, outcomes measured, hospital duration stay, and patient enrolment strategies);

2. Participants' characteristics (age, gender, ethnicity).

Unavailable information from included studies will be sought from corresponding authors by email. Eligible studies will be categorised according to the outcome data they provide (ie, prevalence, mortality, case fatality) and the clinical setting in which the participants are assessed. Any disagreements regarding the inclusion of studies will be resolved by discussion or by consulting a third reviewer. A table of all included studies will be inserted and the reasons for exclusion of studies will be documented.

\section{Quality appraisal of included studies}

The methodological quality of primary studies will be assessed by a quality assessment tool developed by Hoy et $a l^{9}$ and adapted by Werfalli et $a l,{ }^{40}$ which will be applied and adapted, if necessary, to all screened fulltext articles, in order to assess the study quality. The defined questions will be answered and the score of each article will be calculated using this assessment tool. Studies will be graded as low risk, moderate risk and high risk for scores $\leq 5,6-8$ and $>8$, respectively. An independent investigator will be consulted through discussion to reach consensus where there is uncertainty or disagreement between reviewers. An evaluation of the risk of bias will allow for sensitivity analysis.

\section{Data synthesis}

All included studies will be overviewed and presented in two separate tables. The first table will provide details on study quality according to the mentioned tool. The other table will include study design, participants and the characteristics of isolated bacteria.

Our statistical analysis of the primary measures will include two steps: (1) identification of data sources and documenting estimates, and (2) using a random-effects and fixed-effects meta-analysis model to aggregate prevalence estimates and to account for variability between studies, by calculating the overall pooled estimate and the $95 \%$ CI.

Initially, the data will be analysed using a narrative method. Heterogeneity will be evaluated to determine the extent of variation in effect estimates due to heterogeneity rather than chance. The heterogeneity among the primary studies will be evaluated by the forest plots, $\chi^{2}$ test (with significance defined at the $\alpha$-level of 10\%) and $\mathrm{I}^{2}$ statistic.

The prevalence of Gram-negative bacteria in VAP in NICUs from different studies will be pooled through a meta-analysis using STATA V.13 statistical software (Stata Corp. 2013. Stata Statistical Software: Release 13. College Station, Texas, USA: Stata Corp LP). 


\section{Assessment of heterogeneity}

The heterogeneity among the included studies will be assessed using the $\mathrm{I}^{2}$ heterogeneity statistic, reported as a percentage $(\%)$, to determine the extent of variation among the studies. ${ }^{41}$ Categories of heterogeneity will be defined as follows: $\leq 25 \%$ low, $26-50 \%$ moderate, 51$75 \%$ substantial and $76-100 \%$ as considerable, defined by Higgins. Forest plots will also be used to further identify heterogeneity by means of the $\chi^{2}$ test (with significance defined at the $\alpha$-level of $10 \%$ ) and the $\mathrm{I}^{2}$ statistic (where $\geq 50 \%$ indicates substantial heterogeneity).

\section{Sensitivity analysis}

We will implement sensitivity analyses to explore the impacts of methodological quality and sample size on the robustness of review conclusions. Meta-analyses will be repeated after excluding studies with lower methodological quality and studies with sample sizes much larger than those of other studies. Sensitivity analyses will be reported in a summary table, and reviewed conclusions will be interpreted by making comparisons between the two meta-analyses. Any discrepancies or disagreements will be discussed by the reviewers and, if necessary, they will call an independent reviewer to provide clarification.

\section{Subgroup analyses}

Subgroup analyses will be conducted according to the region, gender and isolated Gram-negative bacteria.

\section{Assessment of reporting bias}

The publication bias will be assessed by funnel plots (ie, plots of study results against precision) and Begg's and Egger's tests.

\section{Reporting of this review}

We will make use of flow diagrams to summarise the inclusion criteria and selection process of studies, and also to detail the reasons for exclusion. This systematic review will be reported according to the PRISMA 2009 guidelines. ${ }^{37}$ The search strategy and quality appraisal tool will also be published as online supplementary material documents.

\section{Ethics and dissemination}

Systematic reviews draw on publicly available data and therefore do not require formal ethical review. The findings of this systematic review will be disseminated through peer-reviewed journal publications and conference proceedings.

To the best of our knowledge, there are no systematic reviews that have specifically looked at the prevalence of Gram-negative bacteria in VAP in NICUs. We expect this systematic review will help policymakers and guideline developers in the management of neonates with VAP in NICUs.

Contributors YE, AR and LJ contributed to the concept and study design. AR and LJ developed the search strategies and contributed to the implementation and analysis of the studies. AR contributed to the initial drafting and critical revision and approved the manuscript for submission. AR and LJ revised the manuscript. YE and LJ will also screen potential studies, extract data and assess their quality.

Competing interests None declared.

Provenance and peer review Not commissioned; externally peer reviewed.

Data sharing statement All recorded data from the data extraction process will be available on request to the extent that it is not included in the systematic review article.

Open Access This is an Open Access article distributed in accordance with the Creative Commons Attribution Non Commercial (CC BY-NC 4.0) license, which permits others to distribute, remix, adapt, build upon this work noncommercially, and license their derivative works on different terms, provided the original work is properly cited and the use is non-commercial. See: http:// creativecommons.org/licenses/by-nc/4.0/

\section{REFERENCES}

1. Marik PE. An evidence-based approach to the diagnosis of ventilator-associated pneumonia. Respir Care 2009;54:1446-8.

2. Afjeh SA, Sabzehei MK, Karimi A, et al. Surveillance of ventilatorassociated pneumonia in a neonatal intensive care unit: characteristics, risk factors, and outcome. Arch Iran med 2012;15:568.

3. Gauvin F, Dassa C, Chaïbou M, et al. Ventilator-associated pneumonia in intubated children: comparison of different diagnostic methods. Pediatr Crit Care Med 2003;4:437-43.

4. Gaynes RP, Edwards JR, Jarvis WR, et al. Nosocomial infections among neonates in high-risk nurseries in the United States. Pediatrics 1996;98:357-61.

5. Cook DJ, Reeve BK, Guyatt GH, et al. Stress ulcer prophylaxis in critically III patients: resolving discordant meta-analyses. JAMA 1996;275:308-14.

6. Cordero L, Ayers LW, Miller RR, et al. Surveillance of ventilator-associated pneumonia in very-low-birth-weight infants. $A m$ $J$ Infect Control 2002;30:32-9.

7. Aelami MH, Lotfi M, Zingg $\mathrm{W}$. Ventilator-associated pneumonia in neonates, infants and children. Children 2014;1:16.

8. Apisarnthanarak A, Holzmann-Pazgal G, Hamvas A, et al. Ventilator-associated pneumonia in extremely preterm neonates in a neonatal intensive care unit: characteristics, risk factors, and outcomes. Pediatrics 2003;112:1283-9.

9. Petdachai W. Ventilator-associated pneumonia in a newborn intensive care unit. Southeast Asian J Trop Med Public Health 2004;35:724-9.

10. Foglia E, Meier MD, Elward A. Ventilator-associated pneumonia in neonatal and pediatric intensive care unit patients. Clin Microbiol Rev 2007;20:409-25.

11. Drews MB, Ludwig AC, Leititis JU, et al. Low birth weight and nosocomial infection of neonates in a neonatal intensive care unit. $J$ Hosp Infect 1995;30:65-72.

12. Ford-Jones EL, Mindorff CM, Langley JM, et al. Epidemiologic study of 4684 hospital-acquired infections in pediatric patients. Pediatr Infect Dis J 1989;8:668-75.

13. Hemming VG, Overall JC Jr, Britt MR. Nosocomial infections in a newborn intensive-care unit: results of forty-one months of surveillance. N Engl J Med 1976;294:1310-16.

14. Goldmann DA, Freeman J, Durbin WA. Nosocomial infection and death in a neonatal intensive care unit. $J$ Infect Dis 1983;147:635-41.

15. Craven DE, Barber TW, Steger KA, et al. Nosocomial pneumonia in the 1990s: update of epidemiology and risk factors. Semin Respir Infect 1990;5:157-72.

16. Cook DJ, Walter SD, Cook RJ, et al. Incidence of and risk factors for ventilator-associated pneumonia in critically ill patients. Ann Intern Med 1998:129:433-40.

17. Grohskopf LA, Sinkowitz-Cochran RL, Garrett DO, et al. A national point-prevalence survey of pediatric intensive care unit-acquired infections in the United States. J Pediatr 2002;140:432-8.

18. Babcock HM, Zack JE, Garrison T, et al. Ventilator-associated pneumonia in a multi-hospital system differences in microbiology by location. Infect Control Hosp Epidemiol 2003:24:853-8.

19. Stoll BJ, Hansen NI, Adams-Chapman I, et al. Neurodevelopmental and growth impairment among extremely low-birth-weight infants with neonatal infection. JAMA 2004;292:2357-65.

20. Van der Zwet W, Kaiser A, Van Elburg R, et al. Nosocomial infections in a Dutch neonatal intensive care unit: surveillance study with definitions for infection specifically adapted for neonates. $J$ Hosp Infect 2005;61:300-11.

21. Donowitz LG. Nesocomial infection in neonatal intensive care units. Am J Infect Control 1989;17:250-7. 
22. Harpin VA, Rutter N. Barrier properties of the newborn infant's skin. J Pediatr 1983;102:419-25.

23. Babcock HM, Zack JE, Garrison T, et al. An educational intervention to reduce ventilator-associated pneumonia in an integrated health system: a comparison of effects. Chest J 2004;125:2224-31.

24. Brook I. Treatment of aspiration or tracheostomy-associated pneumonia in neurologically impaired children: effect of antimicrobials effective against anaerobic bacteria. Int $J$ Pediatr Otorhinolaryngol 1996;35:171-7.

25. Cernada M, Brugada M, Golombek S, et al. Ventilator-associated pneumonia in neonatal patients: an update. Neonatology 2014;105:98-107.

26. Zhang DS, Chen C, Zhou W, et al. [The risk factors of ventilator-associated pneumonia in newborn and the changes of isolated pathogens]. Sichuan Da Xue Xue Bao Yi Xue Ban 2013;44:584-7.

27. Baltimore RS. The difficulty of diagnosing ventilator-associated pneumonia. Pediatrics 2003;112:1420-1.

28. Cernada M, Aguar M, Brugada M, et al. Ventilator-associated pneumonia in newborn infants diagnosed with an invasive bronchoalveolar lavage technique: a prospective observational study $^{*}$. Pediatr Crit Care Med 2013;14:55-61.

29. Yuan TM, Chen LH, Yu HM. Risk factors and outcomes for ventilator-associated pneumonia in neonatal intensive care unit patients. J Perinat Med 2007;35:334-8.

30. Zhou Q, Lee SK, Jiang SY, et al. Efficacy of an infection control program in reducing ventilator-associated pneumonia in a Chinese neonatal intensive care unit. Am J Infect Control 2013;41:1059-64.

31. Diouf E, Beye M, Diop NM, et al. [Nosocomial ventilator-associated pneumonia in a tropical intensive care unit]. Dakar medical 2005;51:81-8.
32. Zhang DS, Chen C, Zhou W, et al. [Pathogens and risk factors for ventilator-associated pneumonia in neonates]. Zhongguo Dang Dai Er Ke Za Zhi 2013;15:14-18.

33. Tripathi S, Malik G, Jain A, et al. Study of ventilator associated pneumonia in neonatal intensive care unit: characteristics, risk factors and outcome. Internet $J$ Med Update 2010;5.

34. Badr MA, Ali YF, Albanna EA, et al. Ventilator associated pneumonia in critically-ill neonates admitted to neonatal intensive care unit, Zagazig university hospitals. Iran J Pediatr 2011;21:418-24.

35. Fischer JE, Ramser M, Fanconi S. Use of antibiotics in pediatric intensive care and potential savings. Intensive Care Med 2000;26:959-66.

36. Fischer JE, Allen P, Fanconi S. Delay of extubation in neonates and children after cardiac surgery: impact of ventilator-associated pneumonia. Intensive Care Med 2000;26:942-9.

37. Moher D, Shamseer L, Clarke M, et al. Preferred reporting items for systematic review and meta-analysis protocols (PRISMA-P) 2015 statement. Syst Rev 2015;4:1.

38. Moher D, Liberati A, Tetzlaff J, et al. Preferred reporting items for systematic reviews and meta-analyses: the PRISMA statement. Ann Intern Med 2009;151:264-9.

39. Hoy D, Brooks P, Woolf $A$, et al. Assessing risk of bias in prevalence studies: modification of an existing tool and evidence of interrater agreement. J Clin Epidemiol 2012;65:934-9.

40. Werfalli M, Musekiwa A, Engel ME, et al. The prevalence of type 2 diabetes mellitus among older people in Africa: a systematic review study protocol. BMJ Open 2014;4:e004747.

41. Deeks JJ, Higgins J, Altman DG. Analysing data and undertaking meta-analyses. In: Higgins JPT, Green S, eds. Cochrane handbook for systematic reviews of interventions. Cochrane book series, 2008:243-96. 